\title{
Variations
}

Variations Revue internationale de théorie critique

16 | 2012

Tahrir is here !

\section{Philosophie carcérale}

\section{Alain Guyard}

\section{OpenEdition}

Journals

Édition électronique

URL : http://journals.openedition.org/variations/200

DOI : 10.4000/variations.200

ISSN : 1968-3960

\section{Éditeur}

Les amis de Variations

\section{Édition imprimée}

Date de publication : 16 janvier 2012

\section{Référence électronique}

Alain Guyard, «Philosophie carcérale », Variations [En ligne], 16 | 2012, mis en ligne le 20 février 2012, consulté le 10 décembre 2020. URL : http://journals.openedition.org/variations/200 ; DOI : https:// doi.org/10.4000/variations. 200

Ce document a été généré automatiquement le 10 décembre 2020.

Les ami•e•s de Variations 


\title{
Philosophie carcérale
}

\author{
Alain Guyard
}

\section{NOTE DE L'ÉDITEUR}

Première publication sur www.theoriecritique.com, « Tahrir is here ! », 16 janvier 2012, pp. 93-105

\section{Le détenu sans intérêt}

1 L'association de la philosophie à l'univers carcéral ne peut pas ne pas faire penser à l'héritage foucaldien et notamment, sur le plan théorique à l'un de ses textes majeurs Surveiller et punir ${ }^{1}$, et sur le plan pratique à sa lutte contre les QHS² dans les années 1975 et suivantes. De là, très souvent l'assimilation de mon travail ${ }^{3}$ - professeur de philosophie travaillant en détention - à une sorte de mise en pratique de la philosophie de Michel Foucault.

2 L'objet de cet article est de clarifier cette relation. Même si nul ne peut faire l'impasse sur la pensée de Michel Foucault, à plus forte raison s'il a une pratique professionnelle qui le lie au monde de la prison, ma propre expérience et les réflexions qu'elle a induites m'ont amené à prendre progressivement des distances avec l'auteur de Surveiller et Punir. Cela vient sans doute de ce que je ne suis pas un philosophe qui pense la prison à travers une généalogie des processus politiques qui s'y inscrivent, mais parce que je suis un professeur de philosophie qui travaille en prison. Et je travaille moins avec des concepts qu'avec des prisonniers. C'est au cours de leur fréquentation que j'ai été amené, progressivement, à m'écarter des analyses foucaldiennes. Qu'on se souvienne d'une interview donnée par Michel Foucault deux mois seulement après l'ouverture des $\mathrm{QHS}^{4}$ :

En fait, je ne m'intéresse pas au détenu comme personne. Je m'intéresse aux tactiques et aux stratégies de pouvoir qui sous-tendent cette institution paradoxale, à la fois toujours critiquée et toujours renaissante, qu'est la prison... Ce qui doit servir de fil directeur à l'analyse, ce sont des rapports de stratégie, étant entendu que la stratégie ou la tactique du pouvoir va laisser des marques sur le corps des 
individus, tout comme une guerre laisse des cicatrices sur le corps des combattants.

Mais ce n'est pas la cicatrice qui vous permettra de remonter le fil de la stratégie.

Sans doute ma faiblesse en tant que "philosophe » tient-elle au fait que je ne parviens absolument pas à faire abstraction des personnes. Et si, pour être foucaldien, il faut être capable de se détourner des détenus et de leurs cicatrices, sans doute alors suis-je trop fleur bleue ou pas assez intellectuel pour rejoindre l'écurie des penseurs foucaldiens. En tout cas, il se joue, pour moi, dans le menu détail, dans les cicatrices et les visages marqués, dans le café que l'on boit ensemble au petit matin ou le regard qui se dérobe, dans les manières de se tenir ou de s'avachir, de se taire ou d'écrire, de parler ou de lire, il se joue quelque chose de personnel, d'irremplaçable et auquel je ne parviens pas à me soustraire. La divergence principale qui me retient donc d'adhérer à Foucault tient sans doute en cela que, venant des contreforts de la phénoménologie puis ayant délaissé la philosophie pour lui préférer l'écriture romanesque, j'ai toujours préféré la fréquentation des hommes de troupe à celle des États-majors. Je n'aime guère les hauts gradés qui pensent la bataille pourvu que la guerre et ses stigmates ne parviennent aux généraux que sous la forme d'une idée.

4 Aux fondements de la philosophie foucaldienne se trouvent les notions de biopouvoir, de microphysique du pouvoir et de norme. L'espace de l'enfermement serait ce lieu où se fabriquent des délinquants; ce serait le lieu d'une industrialisation extensive de l'assimilation des normes du milieu par des individus dont toute la sensibilité, la subjectivité, le corps même sont reconstruits par la microphysique du pouvoir. Une telle normativité est instruite par la discipline des processus subtils qui font appel à des dressages indirects de la corporéité de la part d'une institution qui se soucie moins de tuer que de re-dresser. La colonne vertébrale de la pensée foucaldienne sur la prison reste assurément Surveiller et Punir, qui veut débusquer dans l'entreprise carcérale moderne une véritable entreprise d'orthopédie sociale, une tentative pour quadriller, contrôler, mesurer, dresser les individus, les rendre à la fois «dociles et utiles ». Au début de son ouvrage, M. Foucault annonce quatre règles générales qui vont déterminer ses travaux : il s'agit pour lui de considérer la punition comme une fonction sociale complexe, dans la perspective d'une « tactique politique », d'une « technologie du pouvoir » qui s'empare du corps pour l'investir des « rapports de pouvoir $»^{5}$. Les dernières lignes du texte expliquent qu'il ne faut plus lire dans la prison «le fonctionnement unitaire d'un appareil ou d'une institution, mais la nécessité d'un combat et les règles d'une stratégie $»^{6}$. On voit sur quel terrain se place Foucault : il s'agit pour lui de déterritorialiser le judiciaire et le carcéral. «L'univers de la prison est ordinairement le lieu dans lequel on enferme ceux qui ont commis une faute légale ou morale. Le judiciaire punit toute désobéissance à la loi, par conséquent se contente de juger formellement sur le plan légal, et n'intervient pas par conséquent sur le plan moral. Libre à chacun d'avoir sa morale propre et de penser ce qu'il veut, pourvu qu'il se soumette à la légalité qui règle les relations dans le monde commun ", voilà en quelque sorte ce que serait le discours le plus ordinaire. Or Foucault montrera que dans les sociétés disciplinaires, on exige du détenu qu'il fasse retour sur sa peine, qu'il s'interroge sur elle et l'intègre aussi sur le plan de son intimité psychique et spirituelle. D'où la multiplication des psychologues, psychiatres et évaluateurs de tout poil dont la fonction sociale est de s'assurer que le détenu a bien compris le «sens de sa peine ${ }^{7}$. Ainsi, moralité et légalité sont confondues dans une même opération pour se détacher progressivement de tout rapport à la légitimité. Car si on entend mettre aux arrêts les détenus, comme s'ils avaient une vie arrêtée, mise entre parenthèse, Foucault 
traite la question en des termes techno-militaires de rapports de pouvoir qui n'en finissent pas de s'exercer. Le temps mort de l'incarcération n'est surtout pas un temps où le détenu est laissé à l'isolement, retranché hors du monde des hommes. Bien au contraire, on mobilise autour de lui des forces impressionnantes, des stratégies, des pouvoirs complexes dont la fonction est justement de le plier à de nouvelles normes, jusqu'à reconstruire en lui une nouvelle sensibilité et une nouvelle moralité. Foucault déplace donc le regard que l'on porte sur le détenu. Il n'est plus l'homme seul face à sa conscience, il est le cobaye d'une action politique fine qui vise à arraisonner son âme. Il n'y a rien d'autre que cette tactique de conquête militaire qui s'empare de la sensibilité des hommes pour en faire un nouveau champ de bataille. Il convient donc de penser la détention et ce qui s'y passe comme le lieu d'une pure stratégie de conquête des pouvoirs.

Il convient peut-être, pour s'en convaincre, de faire retour sur une connivence intellectuelle intéressante entre Foucault et Vergès. Dans la préface au livre de J. Vergès, De la stratégie judiciaire ${ }^{8}$, M. Foucault explore avec l'auteur les nouvelles stratégies de rupture judiciaire. On voit qu'ici encore l'univers judiciaire, comme l'univers carcéral, sont pensés tous deux en des termes de stratégie de conquête, de tactique militaire où il convient de monter aux extrêmes (pour reprendre une terminologie de Von Clausewitz) pour engager un rapport de pouvoir, de force avec l'ennemi. Cette stratégie, cette tactique militaire sont inséparables d'une réflexion élargie sur la guerre ${ }^{9}$ qui doit irriguer non seulement le tribunal ou la cellule du condamné, mais jusqu'à la vie même, dans sa dimension la plus ordinaire :

M. Foucault: Votre livre a été élaboré et écrit dans une conjoncture historique déterminée et, même si dans son projet il débordait largement le cadre de la guerre d'Algérie, cet événement y est encore très présent et commande sans doute une part de vos analyses. Ne pensez-vous pas que le développement pratique d'une nouvelle stratégie judiciaire impliquerait un travail d'analyse et de critique globale $\mathrm{du}$ fonctionnement judiciaire actuel et comment pensez-vous que l'on pourrait mener collectivement ce travail?

J. Vergès : Ce qui distingue la rupture, aujourd'hui, c'est qu'elle n'est plus le fait d'un petit nombre dans des circonstances exceptionnelles, mais d'un grand nombre à travers les mille et un problème de la vie quotidienne [...] Cela prouve que la stratégie judiciaire... n'est plus seulement l'affaire des gens de robe mais des gens en jeans [...] Ma loi est d'être contre les lois parce qu'elles prétendent arrêter l'histoire, ma morale est d'être contre les morales parce qu'elles prétendent figer la vie.

\section{Le détenu comme surhomme}

6 La conséquence sur le statut du détenu apparaît dans un texte qui peut sembler mineurmais le diable est souvent dans les détails. Il s'agit d'une préface qu'il donna à un recueil de témoignages de prisonniers américains. Cette préface a paru l'année 1975, cette année d'intense activité théorique et pratique de Foucault autour de la prison. Elle me paraît par conséquent d'autant plus importante qu'elle n'est pas un texte connexe et périphérique, mais qu'elle se place en plein dans le moment où l'activité intellectuelle et revendicative de Foucault est sans doute à son acmé dans la question carcérale. Et cependant le texte est mineur, il tient en cinq pages, et surtout, il introduit à un document qui peut sembler aux antipodes de la méthode foucaldienne. En effet, l'auteur du livre est un journaliste qui visite les prisons texanes et rapporte des témoignages de prisonniers, ces « Pantagruels de la délinquance » comme les appelle Foucault et qui sont, pêle-mêle, Margot la Folle, le 
travesti braqueur, Bob, Ray, la Grosse Sal, le carambouilleur Websler, Bebop le toxico, slim le tricheur aux jeux de dés et Maxwell qui s'est fait baiser un nombre incalculable de fois. Voilà qui dénote chez notre philosophe tellement détaché des personnes incarcérées et peu soucieux de leurs "cicatrices». Et cependant le voilà pris au piège de leur dimension autobiographique, de "la "geste" ${ }^{10}$, de leur "récit», le voilà saisi par «l'accumulation, le festin, l'orgie de délits, la surcharge, la fiction sans doute, l'overdose des faits...»

7 Comment Foucault va-t-il donc lire et comprendre l'épaisseur de l'existentiel carcéral ? Foucault entend se séparer du double discours de la « gauche » bien pensante à l'égard du prisonnier. Il récuse le discours victimaire et misérabiliste, issu d'un certain marxisme, ne voyant dans les délinquants incarcérés des "produits de la société ». Mais il récuse également l'approche gauchiste qui voit dans le (la ?) geste de la délinquance « la vraie guerre sociale » engagée par des éléments conscients politiquement. Il conteste donc ce qu'il appelle « le délinquant-victime et la délinquance-rupture ». Que reste-t-il alors pour dépeindre le délinquant?

Et du coup apparaît la chose scandaleuse, l'intolérable vérité qu'il faut étouffer en faisant disparaître définitivement le coupable : c'est que tout avait été aménagé, du haut en bas du système, pour que fonctionnent les illégalismes et que les délinquances les plus profitables se multiplient. [...] Le type du scandale «américain » c'est que... l'homme le plus puissant du monde... que son pouvoir se soit exercé et n'ait pu s'exercer quotidiennement que par la délinquance la plus ordinaire. Finie cette criminalité individuelle et totale des rois shakespeariens qui les entourait d'une aura monstrueuse et d'une certaine façon les sacralisait. On est, depuis longtemps, à l'âge où le fonctionnement du pouvoir et la gestion des illégalismes ont partie liées.

8 Il faut prendre la peine de mesurer la portée de ces quelques lignes. Le projet de Foucault reste toujours le même : faire « disparaître définitivement le coupable », et par extension dépersonnaliser le rapport au détenu existentiel. Comment s'y prendre ? En engloutissant pour ainsi dire le geste délinquant dans la somme de toutes les pratiques politiques qui recourent elles-aussi, pour exister, à la délinquance et à la transgression. On retrouve ici le Foucault familier, celui qui veut penser la société et la politique à travers le seul prisme de rapport de force militaro-stratégique. Président et dealer participent à un même jeu, disparaissent en tant que personnes sur le même plateau d'un même Kriegspiel. La responsabilité morale ( faire disparaître le coupable ») s'évanouit du même coup, et les actions de l'élu à la magistrature suprême comme celles du braqueur travesti sont strictement dé-moralisées. N'importe quel homme en vaut un autre : bourreau et victime, innocent et coupable, ce sont des catégories surannées et inopérantes si l'on veut mesurer ce qui se passe dans les prisons. Il n'y a pas, dedans, des innocents qui se battent pour être reconnus ou des criminels qui s'enfoncent dans le crime ; et il n'y a pas dehors, des chics types et des salauds. Il n'y a que des rapports de force qui amenuisent la puissance des uns et accroissent celle des autres.

9 Et pourtant on ne peut pas comprendre cette perspective dépersonnalisatrice, que j'ai appelé militaro-stratégique sans s'arrêter aux images héroïques mises en scène par Foucault lui-même. Comme si le refoulé revenait en force, non seulement Foucault ne parvient pas complètement à supprimer, à faire disparaitre la présence humaine, mais il la fait resurgir sous des figures sur-humaines, classiques et monumentales. D'un côté la " geste », la « fiction », « l'overdose de faits », « l'orgie » de ces nouveaux « Pantagruels » que sont les prisonniers texans. De l'autre, des « rois shakespeariens » ratés, puisque leur 
criminalité est diluée dans le collectif, qu'elle est relative, qui ne sont plus des monstres ni des êtres sacrés.

10 C'est là, je pense, que se trouve le nœud gordien et le point aveugle de la pensée foucaldienne à propos de la délinquance. Dans une première strate, celle de la microphysique du biopouvoir à l'exercice dans les sociétés disciplinaires, plus rien ne distingue le délinquant de l'honnête citoyen - ou, pour dire comme Vergès, le résistant du terroriste. Le positionnement moral des individus est évacué au bénéfice du positionnement militaire d'unités sur la carte d'Etat-major social du général Foucault. Mais si l'on s'applique à faire ce que Foucault entend faire aux autres, c'est-à-dire cette généalogie du savoir héritée de Nietzsche, on découvre une seconde strate. Ici, le délinquant a cela de plus par rapport à celui qui exerce un pouvoir politique officiel, qu'il est le héros d'une fiction et d'une geste, un Pantagruel (étymologiquement "tout à la gueule ») jusque dans «l'orgie ». Figure profondément nietzschéenne, viscéralement dionysiaque, le délinquant foucaldien est par delà bien et mal parce qu'il est celui qui synthétise les vertus de la fête païenne et des puissances chtoniennes. Son «effet politique ne peut être dissocié du rire énorme qui traverse tous ces récits $»^{11}$. Dans la cosmologie nietzschéenne, le rire, vertu de Dionysos, fera en effet du délinquant un monstre sacré, un guerrier supérieur se moquant des petites tragédies de l'existence ${ }^{12}$ :

Qui de vous peut en même temps rire et être élevé?

Celui qui plane sur les plus hautes montagnes se rit de toutes les tragédies de la scène et de la vie.

Courageux, insoucieux, moqueur, violent - ainsi nous veut la sagesse: elle est femme et ne peut aimer qu'un guerrier.

C'est là l'erreur des princes de ce monde : ce sont des rois sortis des tragédies de Shakespeare, mais des rois ratés parce qu'il leur a manqué la dimension dionysiaque dont les délinquants savent faire preuve. Il leur a manqué cette saine vitalité, cette insouciance violente et supérieure dont seuls les «orgiaques de la délinquance » sont capables.

La vision foucaldienne est donc bien double. Elle est en premier lieu politique et néantise l'individu délinquant pour l'engloutir dans des rapports de force physico-militaire. A ce titre, rien ne distingue le délinquant de l'homme ordinaire. Elle est en second lieu métaphysique, et consacre le délinquant comme "surhomme", seul capable de styliser son existence au rang d'une geste dionysiaque. Ici, il devient un modèle.

\section{Le détenu comme modèle}

Il revient à Christopher Lasch ${ }^{13}$ d'avoir montré en quoi nos élites politiques et culturelles partagent toutes ce culte de ceux que j'appelle en reprenant l'idée de Foucault les "orgiaques de la délinquance». C'est-à-dire que les pratiques de subversion, de contestation, les pratiques dionysiaque de la fête barbare, de la désinhibition, le renversement des normes, des règles, toutes ces caractéristiques qui étaient jusqu'aux années 1950 la marque de fabrique d'une certaine catégorie sociale, une sorte de lumpenproletariat échappant à la sphère de la production et vivant en parasite sur le système - classe d'ailleurs plus fantasmée qu'objective, toutes ces caractéristiques sont devenues celles des parvenus, de tous ceux qui convoitent, possèdent ou défendent un pouvoir politique, économique, culturel ou médiatique (c'est maintenant la même chose) sur le reste de leurs semblables. 
Naguère, c'était la "révolte des masses " qui était considérée comme la menace contre l'ordre social et la tradition civilisatrice de la culture occidentale. De nos jours, cependant, la menace principale semble provenir de ceux qui sont au sommet de la hiérarchie sociale, et non pas des masses. Ce remarquable retournement dans l'Histoire confond nos attentes quant au cours qu'elle était censée prendre et remet en question des présuppositions déjà établies depuis longtemps [...].

$\mathrm{Ma}$ thèse est que toutes ces attitudes mentales [i.e. révolte et menace] sont davantage caractéristiques aujourd'hui des niveaux supérieurs de la société que des niveaux inférieurs ou médians.

Cette perspective de «racaillisation » des élites ${ }^{14}$ va donc de paire avec une glorification de la doxa foucaldienne. Au fur et à mesure que s'étend le règne de la transgression et de la subvention allouée à la subversion, le modèle délinquo-orgiaque fait florès et les classes sociales qui ont le pouvoir symbolique et le capital culturel s'en entichent.

De là, très certainement, le contresens sur mon travail. Il n'est pas question pour moi d'aller au contact avec la population carcérale et de la faire philosopher parce qu'elle serait en quelque sorte dépositaire d'un instinct très sûr de la subversion dionysiaque. Je ne descends pas en détention comme on part en pèlerinage pour y recueillir la parole et la « geste» de ces monstres admirables qui savent se rire de tout tragique de l'existence. Seulement voilà, ceux qui me rencontrent et m'interrogent sur ma pratique sont souvent issus de cette catégorie des "élites révoltées", toutes fascinées qu'elles sont par la dégaine du mauvais garçon à qui on ne la fait pas et qui pourrait en apprendre au premier intellectuel venu. Ces a priori idéologiques, on voit qu'ils dérivent des leçons de Foucault et du Zeitgeist qui laisse la parole aux hommes de la «culture prison » pourvu qu'ils revendiquent, même indirectement, le braquage comme geste esthétique ${ }^{15}$.

\section{De pauvres gens}

Mais alors qu'y fais-je ? J'y fréquente d'abord des gens que Foucault n'a pas connu, parce qu'il les a évités et parce que la prison a changé. Ce sont des gens ordinaires, des petites gens, guère orgiaque, encore moins dionysiaque. Ce sont des trafiquants aux petits pieds issus des quartiers les plus pauvres et les plus immigrés, petits artisans du néocapitalisme maximisant la plus-value dans l'import-export de produits vendus à des moins pauvres et moins immigrés. Ce sont des gens caparaçonnés dans les certitudes d'un Islam simpliste qui les fait haïr le paganisme orgiaque en public. Ce sont des artisans de souche ayant conduit sans permis ou avec défaut d'assurance. Ce sont des papas qui boivent et qui tapent la maman. Ce sont des malades mentaux, laissés sans soin depuis que les hôpitaux psychiatriques ferment leurs unités, ou bien des jeunes adultes dans une misère si noire, tellement désocialisés et sans le secours des services sociaux qui ferment à leur tour, qu'ils se réjouissent de trouver dans la prison le « foyer » qui leur fait défaut, dehors. Ce sont des chèques volés. Des trafics à l'assurance. Des combines à la carte bleue. Une bagarre à la sortie d'un bistro. Un mot de travers à un gendarme, parce que l'alcool, l'épuisement, le dégoût... Interrogés sur leur vision du monde, ils sont à mille lieues de vanter un imaginaire de la révolte ou de la subversion. Combinards bancals et émouvants dans leur maladresse, ils ne rêvent à rien d'autre qu'à s'insérer dans le modèle de consommation capitaliste. Tout au plus, parfois, en font-ils la critique, mais c'est pour vanter à regret les mérites de la famille, de la religion ou du redressement par l'armée. Ce sont des pauvres gens. 
Il est bien difficile de foucaldiser avec ces gens-là... D'abord leurs cicatrices sont trop visibles et il est impossible de ne pas les voir. Les dents manquent, la peau est marquée de maladies, l'accent dénote le quartier juste de l'autre côté du périphérique, le corps n'est pas habitué à s'asseoir longuement, la parole tourne et cherche en désordre... Et puis, avec eux, impossible de valoriser l'orgiaque, d'autant plus qu'ils appellent tous de leurs vœux des régulations politiques, psychosociales ou thérapeutiques. Enfin, les inscrire dans des systèmes stratégiques de conflictualité est leur fait grand honneur, eux qui sont pour leur plus grande part les soutiens inconditionnels du système qui les broie et les exploite ${ }^{16}$.

\section{Une douce stupéfaction}

17 Quoi faire avec eux, et quelle est la place allouée à la philosophie dans la relation que je tisse avec eux? Il faut revenir à trois outils, dont je me sers essentiellement dans ma pratique de philosophie carcérale, et qui sont la narration philosophique, l'atelier d'écriture et la macro-rhétorique.

Qu'est-ce que la narration philosophique? Le public avec lequel je travaille, je m'en suis expliqué plus haut, est majoritairement éloigné des pratiques textuelles. L'illettrisme, la télévision allumée presque en permanence dans les cellules, le niveau scolaire très bas, le vacarme permanent qui est le lot de toutes les prisons, tout cela rend difficile le recueillement physique et spirituel que demande la lecture. En revanche, pour eux, l'oralité tient une grande place. D'abord pour les détenus issus du monde du Maghreb, chez lesquels le souvenir de la place du conteur ou du poète est encore très vif et vaut la plus grande considération. Ensuite pour tous les détenus, qui ont perdu le contact avec une parole qui ne soit pas injonctive ou inquisitoriale, et qui sont vite conquis par la parole narrative parce qu'elle n'exige rien d'eux en terme de subordination ou d'aveu. Ainsi donc, je « raconte » mes philosophes, leur vie ou des situations idéales par lesquelles s'incarnent leur philosophie ${ }^{17}$. Mais je ne me contente pas de les raconter comme s'il s'agissait d'égayer une matinée par des anecdotes piquantes. J'emploie les recours et les méthodes qui sont celles des contes traditionnels: modulation de la voix, usage de ritournelles, fabrication de complicités avec le public, tension et relâchement dramatique, intrigue et dénouement. C'est sur la forme que je joue, autant que sur le fond, puisqu'il s'agit de mettre l'auditoire dans une situation de rêverie attentive, à la fois concentrée et relâchée ${ }^{18}$. Ce relâchement n'est pas qu'une vue de l'esprit. C'est aussi la recherche d'une modification concrète et réelle de l'attitude des détenus, dans leur posture physique, dans l'abandon auquel ils consentent lorsqu'ils ont compris qu'il n'y a pas de ma part une volonté impérialiste de faire pression sur eux par la culture, lorsqu'enfin ils s'abandonnent, en toute confiance, au fil de la narration et se laissent bercer de mots sans craindre de s'y perdre.

Et de même, quelle est la fonction de l'atelier d'écriture philosophique ? Il s'agit de suivre des propositions d'écriture, de s'y contraindre pour assister, stupéfait, à une création qui déborde celui qui en est l'auteur. On n'écrit jamais en le voulant, mais, au contraire, en laissant aller la plume, quitte à griffonner des pages et des pages qui sont comme un échauffement au terme duquel enfin, quelque chose ou quelqu'un écrit, en moi, qui n'est pas ce moi sur lequel j'ai la barre. Les propositions d'écriture prennent appui sur des textes classiques de la littérature dont on peut faire une approche philosophique ${ }^{19}$. Il est donné lecture du passage en question, puis, à charge pour chacun de prendre appui sur ce 
texte pour s'engager dans le processus d'écriture, en suivant des propositions qui sont modelées selon le contexte. Mais l'essentiel n'est pas là, car c'est sur le plan formel que tout se joue. C'est-à-dire qu'on ne peut entrer en sidération philosophique qu'à la condition d'avoir été dérangé, déplacé par la forme même de l'exercice. Le recueillement pendant la narration devant une parole enfin libérée de toute inféodation à des stratégies de pouvoir ${ }^{20}$, de même que la stupéfaction d'assister aux surgissements de mots auxquels on n'avait pas songé, et sur lequel on n'avait projeté aucune intention avant qu'ils n'apparaissent sur le papier, c'est cela la forme, et cela a pour fonction d'altérer doucement, de manière infinitésimale les cadres de la perception ordinaire.

Il s'agit donc de développer une qualité d'écoute chez les détenus, écoute grâce à laquelle c'est toute leur configuration de détenu occasionnel ou chronique qui opère une translation subtile, parfois invisible. Cette écoute est autant de l'autre, lorsque je narre, que de soi, lorsqu'ils écrivent. Qu'écoutent-ils? Une parole insolite, qui n'était pas programmée dans leur existence, et qui advient, contre toute attente. Irruption de la différence de soi d'avec soi, source noire de toute créativité, avènement dans le silence et dans le renoncement au vouloir de ce quelque chose qui est plus que moi mais qui ne me transcende pas. C'est cela même qui peut les mettre en état de stupéfaction douce et les disposer à la posture philosophante.

\section{L'art des incantations}

$21 \mathrm{Au}$ terme de l'exercice, qui prend souvent plus d'une heure, la parole circule dans le groupe et va bon train. Mais de quelle parole s'agit-il ? D'une parole philosophante, bien sûr, en ce que, dans son fond comme dans sa forme, elle a pour fonction de déplacer les bornes existentielles, de modifier les perceptions, de repenser pour les transformer, les rapports à soi, aux autres, au monde. Dans un cas comme dans un autre, ce doux décollement, ce pas de côté opéré par l'oralité ou l'écriture est immédiatement suivi d'un débat à bâtons rompus, selon le mode socratique, dont la fonction est, pourrait-on dire, d'enfoncer le clou et d'acter dans la conscience même le déplacement existentiel goûté, apprécié, intuitivement ressenti auparavant. Pour le dire autrement, la dimension débattante de la macro-rhétorique a moins pour fonction d'exposer un point de vue d'auteur que de prolonger le mouvement qui s'est fait jour à l'orée de la conscience du détenu. L'état que j'ai qualifié de stupéfaction, ou d'étonnement, pour parler comme Aristote, ou de perceptude pour dire comme Lamande, risque très vite d'être enfoui sous des monticules de justifications et de contre-arguments rationnels qui sont autant de stratégies de peur de la nouveauté. La coquille se referme et tous les efforts pour opérer le déplacement existentiel auront été vains. Quiconque a assisté à un débat entre intellectuels sait très bien ce que je veux dire: exposer des arguments est d'abord une manière de refuser de rendre compte de sa propre vie, et l'on pense souvent avec force pour éviter d'avoir à se sentir faible. Ainsi ma stratégie s'apparente-elle plutôt à la «macro-rhétorique» repérée chez Socrate par Rossetti ${ }^{21}$ qui insiste sur le "coté inauthentique du dialoguer platonicien $\|^{22}$. Au risque parfois de la mauvaise foi, le dialogue que j'installe vise moins à faire éclater les toutes-puissantes lumières de la raison universelle - existent-elles d'ailleurs autrement que comme une mauvaise foi à laquelle j'oppose la mienne? - qu'à prolonger l'état de flottement, de state dans les certitudes, d'apesanteur pressentie dans l'écoute orale ou écrite goûtées plus haut. En quoi consiste cette macro-rhétorique ? Non pas à enseigner le vrai, mais à déployer dans 
le langage les conditions d'un «Grand parler $»^{23}$, d'une parole qui débat jusqu'à contraindre le partenaire de débat à ne plus pouvoir penser selon les catégories closes dans lesquelles il évoluait auparavant. Parole ensorcelante dans la bouche de Socrate, elle s'inscrivait dans « l'art des incantations » - et non rationaliste comme veut nous le faire croire toute une tradition universitaire - duquel le jeune Platon dira par la bouche de Socrate ${ }^{24}$ qu'il est

en effet un art qui sait charmer serpents et tarentules, scorpions, toutes les autres bêtes sauvages, et aussi les maladies; mais c'est aussi l'art qui exerce son charme sur les juges, sur les citoyens à l'Assemblée, sur toute sorte de foule et qui sait les apaiser.

\section{Briser la vie unidimensionnelle}

Quand donc on s'extasie sur mon travail en s'imaginant que je suis de ceux qui sacrifient aux culte des héros dionysiaques enchaînés et sublimes, je suis obligé d'opposer un démenti formel et de ruiner la parentèle foucaldienne. Car si je viens philosopher en prison, c'est parce que j'y rencontre des hommes étrangers à Dionysos et à Apollon mais dont la séquestration et la mise au secret les a enfermé dans une clôture si hermétique que ce serait plutôt sous l'égide d'Hermès que je les invite au travail. Hermès, père des fermetures les plus hermétiques mais aussi messager des dieux, et par là, porteur d'une parole libératoire et qui lui reste, malgré tout, étrangère.

On dira que ce travail n'est guère politique, au sens où il n'est pas inscrit dans les stratégies militaro-politiques subversives du biopouvoir foucaldien. Je crois avoir montré qu'aujourd'hui, c'est le mépris structuraliste apporté à la puissance singulière de création, c'est l'adoration par les classes dirigeantes de la posture subversive, c'est la glorification de la prédation qui sont comme par hasard les clefs de la conquête du pouvoir et de la conservation dans les rapports de domination. Pour le dire autrement, au risque de la brutalité, c'est toute la doxa autour de Foucault qui est dorénavant au service de la domination. S'il est nécessaire de lutter contre toutes les aliénations, il est nécessaire de s'inscrire intellectuellement et pratiquement contre la gauche de subversion. Autrement dit : il est nécessaire de restituer aux pauvres gens perdus dans la nuit de l'enfermement, la singularité de leur possibilité créatrice, les mettre en état de recueillir dans le silence de la parole, quelque chose qui advient et brise les canaux étroits de la vie unidimensionnelle.

Pourquoi un tel travail a-t-il particulièrement son efficacité avec les détenus ? Parce que l'oralité ordinaire en prison est terriblement inscrite dans des rapports de force et de domination, beaucoup plus qu'elle ne l'est à l'extérieur. L'impact d'une parole dégagée des stratégies de conquête est d'autant plus important. Et de même, la non-familiarité avec l'écriture permet, lorsque la plume se délie, des surprises et des enchantements qui ne sont guère goûtés par les gens du dehors. Les détenus sont enfermés à double-tour. Ils le sont d'abord dans l'étroitesse de leur cellule de neuf mètres carrés. Ils le sont également dans les déterminations sociales et économiques qui les aliènent à l'idéologie dominante. Le double travail de l'oralité et de l'atelier d'écriture leur offre l'apesanteur et l'instant de grâce de l'écoute stupéfaite. La macro-rhétorique socratique, cet art incantatoire, leur permet d'échapper au moins l'espace d'un instant, à la pesanteur de cet autre enfermement, celui qui fixe les âmes à la prison comme le clou du sorcier fixe les ombres à la croisée des chemins. 


\section{NOTES}

1. M. FOUCAULT, Surveiller et punir. Naissance de la prison, Paris, Gallimard, 1975.

2. Quartier de Haute Sécurité. Quartiers à sécurité renforcée crées la même année que la publication de Surveiller et punir, en mai 1975.

3. J'ai enseigné et continue d'enseigner la philosophie depuis 2003 dans le cadre de Centres Pénitentiaires, que ce soient des Maisons d'Arrêt ou des Centres de Détention. Ce travail s'est effectué tantôt dans le cadre de collaboration avec l'Éducation nationale, tantôt avec celui des Services de Probation et d'Insertion Pénitentiaire, ou bien encore avec le concours des Juges d'Application des Peines, des Associations d'insertion locale ou développant la politique culturelle en milieu carcéral, ou bien encore dans le cadre de stages diplômant et professionnalisant adressés aux détenus dans lesquels j'intervenais à titre de formateur professionnel de philosophie. On le voit, les formes sont variées, changeantes au gré des crédits alloués ou de leur disparition... A chaque dispositif, il m'aura fallu trouver de nouvelles manières de faire de la philosophie. En revanche, mon travail n'a jamais été de préparer des étudiants au baccalauréat ou à des diplômes universitaires. Il a consisté, généralement, à faire des leçons de philosophie suivie de débat soit sur des sujets que je choisissais, soit sur des sujets pour lesquels j'avais été engagé, soit enfin sur des sujets que me proposaient les détenus eux-mêmes. J'ai peu à peu introduit dans ces ateliers philosophiques une bonne part de "narration philosophique ", dont la forme emprunte au conte, et sur lesquels je reviendrai plus tard. Je me suis servi aussi d'ateliers d'écriture. Mais sur tout cela je reviendrai.

4. «Michel Foucault, l'illégalisme et l'art de punir », entretien avec G. Tarrab, La Presse, $\mathrm{n}^{\circ}$ 80, 3 avril 1976, pp. 2 et 23.

5. Ibid, p. 31.

6. Op. cit., p. 360.

7. N. FRISE, Le sens de la peine, Paris

8. M. FOUCAULT, « Préface à la deuxième édition », in J. VERGÈS, De la stratégie judiciaire, Paris, Éd. de Minuit, 1981, pp. 5-13.

9. En l'occurrence sociale, mais à partir des prédicats de la guerre coloniale menée en Algérie par les autorités françaises.

10. Préface, in Bruce JACKSON, Leurs prisons. Autobiographies de prisonniers américains, Paris, Plon, 1975, pp. I-VI.

11. Op. cit., p. IV.

12. F. NIETZSCHE, Ainsi parlait Zarathoustra, I, « Lire et écrire ».

13. C. LASCH, La Révolte des élites et la trahison de la démocratie, 1994.

14. Que dit celui qui déclare passer au karcher la racaille ? Et à qui le dit-il ? Lisons plutôt : «Je pense qu'on se construit en transgressant, que l'on crée toujours en transgressant. Moi-même, j'ai créé mon personnage en transgressant certaines règles de la pensée unique. Je crois en la transgression [...] L'intérêt de la règle, de la limite, de la norme, c'est justement qu'elles permettent la transgression. Car la liberté, c'est de transgresser.» N. SARKOZY, «Dialogue avec Michel Onfray ", Philosophie magazine, avril 2007.

15. Voir par exemple la mise en scène spectacularisée de Redouane FAÏD, l'auteur de Braqueur des cités au grand banditisme, La Manufacture de livres, 2010, dans les émissions de Canal +. Qualifié d'«archétype du beau mec" par son éditeur sur son site internet, l'homme tient certes un discours de condamnation du grand banditisme mais les cadrages bâtissent un propos filmique exactement contraires avec par exemple, des contreplongées sur le « héros » en costume italien 
impeccable, le crâne aussi lisse que sa chemise est immaculée, au milieu des barres de HLM de sa jeunesse... Une bande annonce pour GTA IV... En outre, pur produit du spectacle, Redouane Faïd ne cesse de dire qu'il construit ses braquages en s'inspirant des mises en scène du "cinéma ». Ainsi, fétiche esthétisé, le voyou a quitté depuis longtemps la voie publique qui lui valait son étymologie pour n'être que pure surface et modèle de propagande pour le non-lieu du spectacle.

16. Un souvenir émouvant : la lecture du journal par un braqueur de bijouterie à l'occasion de la future élection de Barack Obama. Le braqueur, jeune homme noir, s'identifia spontanément à Obama. Lisant le programme d'Obama puis celui de son adversaire conservatrice républicaine, pour ne pas dire d'extrême droite, Sarah Palin, le braqueur découvrit au fil des lignes combien il se sentait proche des thèses de cette dernière: il était lui aussi contre l'avortement et l'euthanasie, pour les préceptes de la religion, contre les impôts et pour la libre entreprise, pour le port d'arme, pour la peine de mort, et enfin at least but not at last, contre les homosexuels... On cherchera en vain aujourd'hui des hommes du combat collectif, ou des marxistes comme Charlie Bauer ou James Carr en prison: il n'y en a plus. Car depuis le triomphe de l'individualisme marchandisé, il n'y a plus de bandes chez les bandits.

17. J'utilise ici beaucoup des chries et des apophtegmes rassemblés par Diogène LAËRCE dans sa Vie et doctrine des philosophes illustres. Mais il est possible de prendre appui sur toute une littérature philosophique que l'on peut convertir en histoire à raconter. La Peste, l'Étranger, Huis-Clos, Candide, l'Iliade et l'Odyssée, Candide sont de ces livres-romans dont la dimension métaphysique est évidente.

18. Cet état, il est qualifié de perceptude par J.-L. LAMANDE, in "L'Ombre de la séduction », site internet des États généraux de la psychanalyse, qui l'oppose ainsi à la perception consciente ordinaire. Il procède d'un état ou le système sélectif de reconnaissance perd ses habitudes et ses stéréotypes. Il en découle une altération de la sensorialité, de la corporéité, des humeurs et du langage.

19. Ainsi par exemple de l'Alice de Lewis Carrol, ou bien de passages d'A la recherche du temps perdu qui décrivent ce qui se passe au réveil, de Flaubert qui décrit dans son Éducation sentimentale ce que c'est que la rencontre amoureuse, ou Apollinaire qui dit comment passe le temps dans son Pont Mirabeau, des cas de conscience de Javert chez Hugo, l'amour fusion dans le Mythe de l'androgyne de Platon, etc...

20. Le récit ne rime à proprement parler à rien : il n'apporte rien, ni réduction de peine ni information monnayable dans le cadre de la détention.

21. Livio ROSSETTI, Le Dialogue Socratique. Avant-propos de F. Roustang, Paris, Les Belles lettres, 2011.

22. Cf. F. COSSUTTA et M. NARCY (edts.) La Forme dialogue chez Platon, Jérôme Million, Grenoble, 2001, pp. 99-118.

23. L'expression est de Pierre Clastres.

24. PLATON, Euthydème, 289 e, sq.

\section{AUTEUR}

\section{ALAIN GUYARD}

Professeur de philosophie en milieu carcéral. Il a notamment écrit La zonzon, 2011, Paris, La Dilettante, $288 \mathrm{p}$. 